\title{
Peripubertal orchidectomy transitorily affects age-associated thymic involution in rats
}

V. Pešić ${ }^{1}$, K. Radojević ${ }^{2}$ D. Kosec ${ }^{2}$,

B. Plećaš-Solarović ${ }^{1}$, M. Perišić ${ }^{2}$, I. Pilipović ${ }^{2}$ and G. Leposavić 1,2

\author{
${ }^{1}$ Department of Physiology, Faculty of Pharmacy, University of Belgrade, \\ Belgrade, Serbia \\ "Immunology Research Center "Branislav Janković", Institute of Virology, \\ Vaccines and Sera "Torlak", Belgrade, Serbia
}

\author{
Correspondence \\ G. Leposavić \\ Faculty of Pharmacy \\ 450 Vojvode Stepe \\ 11221 Belgrade \\ Serbia \\ Fax: +381-11-397-2840 \\ E-mail: \\ gordana.leposavic@pharmacy.bg.ac.yu \\ Research supported by project \\ grant No. 145049 from the Ministry \\ of Science and Environmental \\ Protection of the Republic of \\ Serbia. \\ $\ldots \ldots \ldots \ldots \ldots \ldots$
}

Received March 9, 2007

Accepted July 24, 2007

\begin{abstract}
The role of gonadal hormones in induction and, particularly, maintenance/progression of rat thymic involution, which normally starts around puberty, was reassessed by examining the effects of peripubertal orchidectomy on thymic weight and morphometric parameters at different times up to the age of 10 months. Up to 6 months postcastration both thymic weight and cellularity in orchidectomized (Cx) rats were greater than in age-matched control rats, sham $\mathrm{Cx}(\mathrm{Sx})$. The increase in thymic cellularity reflected an increase in thymocyte proliferation rate (the proportion of proliferating cells was $18.6 \pm$ $0.7 \%$ in 2-month-old $\mathrm{Cx}(\mathrm{N}=5)$ vs $13.4 \pm 0.3 \%(\mathrm{~N}=5)$ in age-matched Sx rats) followed by reduced sensitivity to apoptotic signals (apoptotic thymocytes were $9.8 \pm 0.9 \%$ in 2-month-old $\mathrm{Cx}(\mathrm{N}=5)$ vs $15.5 \pm$ $0.3 \%(\mathrm{~N}=5)$ age-matched $\mathrm{Sx}$ rats). However, 9 months post-orchidectomy, neither thymic weight and cellularity nor any of the morphometric parameters analyzed differed between $\mathrm{Cx}$ and control rats. The reduction of thymic cellularity in $\mathrm{Cx}$ rats to control values may be related to increased sensitivity of their thymocytes to apoptotic signals in culture (72.6 $\pm 1.2 \%$ in 10-month-old vs $9.8 \pm 0.9 \%$ in 2-month-old $\mathrm{Cx}$ rats) followed by reduced responsiveness to proliferative stimuli (14.1 $\pm 0.2 \%$ in 10-month-old vs $18.6 \pm 0.7 \%$ in 2-month-old Cx rats). Thus, the study indicates that the effects of peripubertal orchidectomy on thymic weight and cellularity, as well as on the main morphometric indices, are long-lasting but not permanent, i.e., that removal of the testes can only postpone but not prevent age-related organ atrophy and consequently functional deterioration of the immune system.
\end{abstract}

Key words

- Rat thymus

- Aging

- Orchidectomy

- Stereological analysis

- Thymocyte proliferation

- Thymocyte apoptosis .....................

\section{Introduction}

Thymic involution is one of the most clear-cut effects of aging on the immune system of both laboratory animals and humans. In rodents, there is a sharp increase in immunological activity after birth, reaching a peak at sexual maturity (1). Thereafter, a gradual decline in immune function occurs. Changes in thymus weight and structure, as well as in its capacity to produce T lymphocytes, also become evident around puberty 
(1-3). Thus, as the thymus involutes, the output of newly generated $\mathrm{T}$ cells declines, with a reduction in the level of naive $T$ cells in the periphery. These changes have been related to the age-associated decline in function of the immune system, leading to an increased frequency of specific infections, malignant diseases and autoimmune disorders in old individuals (4). The fact that thymic involution is associated with immunosenescence and its various associated diseases has prompted many studies aimed at understanding the causes and mechanisms of thymic degeneration which may ultimately lead to the possibility of manipulation. This might not only prolong life, but also improve its quality, due to the absence of severe associated pathologies.

Since thymic involution in rodents becomes obvious at the same time as the increase in production of gonadal steroids, a causal link between increasing sex steroid level and age-related thymic atrophy has been suggested $(1,2)$. This notion is supported by data indicating that androgens in males modulate both thymic weight and $\mathrm{T}$ cell maturation by acting either directly on the thymocytes (5) or indirectly on thymic epithelial cells (TEC) (6) supporting T-cell differentiation/maturation. Namely, substantial alterations in the composition of thymocyte subsets at different stages of maturation (delineated by expression of the major differentiation antigens CD4, CD8, T-cell receptor), which are localized in clearly defined thymic compartments, have been observed in animals subjected to gonadectomy $(7,8)$. A putative role for sex steroids in the induction of thymic involution is also supported by numerous experiments showing reversal of orchidectomy-induced thymic hypertrophy by androgen replacement (7). However, the long-term commonly held view that gonadal hormones have a pivotal role in the induction and maintenance of thymic involution has recently been disputed. Namely, thymic involution in humans is pro- posed to be a sex steroid-independent event (9), while lack of gonadal hormones was shown not to produce a delay in age-associated thymic involution in hypogonadal HPG/ Bm-hpg/hpg mice (10). The obvious discrepancy between these and numerous earlier studies may be reconciled by the fact that the role of sex steroids in thymic involution was estimated by exploring the effects of gonadal hormone deprivation/administration for not more than a few months. Therefore, the present study was undertaken to reassess the putative role of gonadal hormones in the induction and, particularly, in the maintenance/progression of age-associated thymic atrophy in rats. To this end, we followed the effects of peripubertal orchidectomy on the rat thymus for up to 9 months after surgery. Since it has been shown that the functional capacity of the aged residual thymus correlates with anatomical measurements of so-called true thymic tissue (4), we subjected this thymic component (i.e., cortical and medullary lymphoid tissue) from both orchidectomized $(\mathrm{Cx})$ and control rats to a tentative morphometric analysis. Additionally, since changes in thymic cellularity mainly reflect alterations in the main homeostatic processes, i.e., apoptosis and proliferation, thymocytes from $\mathrm{Cx}$ and control rats were examined for their sensitivity to both apoptotic and proliferative signaling.

\section{Material and Methods}

\section{Animals}

Male inbred AO rats were maintained under a standard 12-h photoperiod, at $21 \pm$ $2^{\circ} \mathrm{C}$, with free access to food and water. At the age of 30 days, the rats were submitted to bilateral $\mathrm{Cx}$ or sham $\mathrm{Cx}(\mathrm{Sx})$ as previously described (8). All animal experimentation was conducted according to local ethical guidelines and was approved by our Institutional Animal Care and Use Committee. 
One, 3, 6, or 9 months after orchidectomy, the rats were killed under ether anesthesia by exsanguination. Thirty-day-old intact rats, as well as 2-, 4-, 7-, and 10-monthold $\mathrm{Sx}$ and non-operated rats were included in the study as controls. All groups consisted of 5 animals. Each thymus was aseptically isolated, trimmed of all excess body fat and gently blotted on gauze to remove excess blood. The two lobes were divided and weighed individually. The right lobe was used for preparation of thymocyte single cell suspensions and the left lobe was fixed in Bouin's solution and further processed for morphometric analysis.

\section{Computer-assisted morphometric measurements}

Thymic specimens fixed in Bouin's solution and embedded in paraffin were serially cut into $5-\mu \mathrm{m}$ thick sections. Every 40th section (approximately 20-30 sections per organ) was stained with hematoxylin and eosin. Morphometric measurements were made by a point counting method as previously described (11) using an Olympus BX50 microscope and image analysis software (Micro Image, Version 4.0, Olympus Optical Co. GmbH, Hamburg, Germany). The test areas were randomly chosen and each image, acquired using a digital camera, was saved, overlaid with the corresponding grid and analyzed.

Absolute volumes of the main thymic compartments, i.e., cortex, medulla and subcapsular/interlobular connective/adipose tissue, were estimated from the volume of the processed and embedded organ and volume density $(\mathrm{Vv})$ of the corresponding compartment. The relative amount of thymic tissue shrinking during processing and embedding (approximately 34\%) in all groups was determined stereologically, as described earlier (11). Thus, all stereological data refer to fixed thymic tissue. Each thymic compartment $\mathrm{Vv}$ was determined at 40X magnifica- tion using an orthogonal test grid with 130 points, and by dividing the number of test points hitting the analyzed structure by the total number of test points falling on the organ. The overall number of test areas was 100 per animal.

The total number of thymocytes in the thymic compartment was calculated from the numerical density (Nv) of thymocytes, as the number of cells per volume unit, and the absolute volume of that compartment. When calculating the overall number of cortical thymocytes it was taken into consideration that: i) two cortical subcompartments, i.e., outer and deep cortex (12) can be delineated morphologically and functionally, ii) three quarters of cortical thymocytes are situated in the deep cortex (13). The Nv of thymocytes was estimated at immersion magnification using a grid that corresponds to the multipurpose $\mathrm{M}_{42}$ test-system. The test grid was placed randomly, but positioned parallel to and just touching the capsule for the outer cortex and the corticomedullary junction for the deep cortex analysis, respectively. To estimate the $\mathrm{Nv}$ of medullary thymocytes, the grid was placed randomly throughout the medulla. For each thymic compartment 60 test areas per animal were measured.

\section{Preparation of thymocyte suspensions}

Thymocyte suspensions were prepared by gently grinding the thymic tissue on a sterile $60-\mu \mathrm{m}$ sieve screen in complete RPMI1640 medium. These cell suspensions were washed in ice-cold complete RPMI-1640 medium, and the number of cells in each of them was enumerated using an improved Neubauer hemocytometer. As expected (14), the total number of thymocytes/thymus thus estimated did not significantly differ from the corresponding value measured morphometrically. The viability of cell preparations (as determined by Trypan blue exclusion) was routinely greater than $95 \%$. 
Analysis of cycling cells

Propidium iodide binding to DNA was utilized to identify cells in active phases of the cell cycle. As previously described (14), $100 \mu \mathrm{L}$ RPMI 1640 with $5 \mu \mathrm{g} / \mathrm{mL}$ concanavalin A (ConA, Sigma-Aldrich Chemie, Taufkirchen, Germany) was added to $100 \mu \mathrm{L}$ of a cell suspension containing $2.5 \times 10^{5}$ thymocytes dispersed into plastic 96-well plates (Nunc A/S, Roskilde, Denmark) to attain a final ConA concentration of $2.5 \mu \mathrm{g} / \mathrm{mL}$. All cultures were run in triplicate. Cells were harvested after $48 \mathrm{~h}$ of culture. A total of 0.5 x $10^{6}$ cells was fixed by the addition of 400 $\mu \mathrm{L}$ ice-cold absolute ethanol, on ice, for 30 min. The cells were then centrifuged at 300 $g$ for $10 \mathrm{~min}$ and the supernatant was decanted. After the addition of $1 \mathrm{mg} / \mathrm{mL} \mathrm{RNAse}$ 1A (Sigma-Aldrich Chemie) in $250 \mu \mathrm{L}$ phosphate-buffered saline, $\mathrm{pH} 7.4$, to the pellet and vortexing, the samples were incubated in a water bath at $37^{\circ} \mathrm{C}$ for $20 \mathrm{~min}$. Propidium iodide (Sigma-Aldrich Chemie) at 20 $\mu \mathrm{g} / \mathrm{mL}$ in $250 \mu \mathrm{L}$ was then added and the samples were gently mixed and incubated for at least $10 \mathrm{~min}$ at room temperature. Next, the cells were passed through a fine nylon mesh and analyzed with a FACScan flow cytometer (Becton Dickinson, Mountain View, CA, USA). The gate was set for singlet populations so that doublets and higher order cell aggregates would be excluded from DNA analysis.

\section{Detection of apoptotic thymocytes}

Since apoptotic thymocytes are normally rapidly eliminated by phagocytes in vivo, the relative number of apoptotic cells was quantified after $18 \mathrm{~h}$ of cultivation, as previously suggested (15). A $100-\mu \mathrm{L}$ aliquot of the cell suspension (at a concentration of 2.5 x $10^{6} / \mathrm{mL}$ in RPMI 1640 complete medium) was added to each well of a 96-well flatbottom plate (Nunc A/S). The cells were incubated for $18 \mathrm{~h}$ at $37^{\circ} \mathrm{C}$ in a $5 \% \mathrm{CO}_{2}$ humidified atmosphere. Apoptotic thymocytes were detected using merocyanine 540 (MC540). Similarly to annexin-V, this lipophilic dye stains an altered packing order of phospholipids in the outer leaflet of the apoptotic cell plasma membrane. Thus, the percentage of apoptotic cells labeled with MC540 is shown to be equivalent to that obtained by annexin-V staining (16).

The procedure described by Mower and collaborators (17) was used for MC540 staining. All samples were analyzed on the same day with the FACScan flow cytometer (Becton-Dickinson) using CellQuest Software (Becton-Dickinson).

\section{Statistical analysis}

Data are reported as means \pm SEM. Thymic weight and stereological parameters from Sx and non-operated rats of the same age were compared by the Mann-Whitney Utest. Since none of the values of the parameters analyzed differed significantly between Sx and non-operated rats of the same age, the data for these two groups were pooled and presented as one group ( $\mathrm{Sx})$ in all graphs. The differences among groups of different ages were tested by non-parametric Kruskal-Wallis one-way analysis of variance followed by the Mann-Whitney U-test whenever differences were found. The SPSS 10.0 software for Windows was used for statistical analysis.

\section{Results}

\section{Histology}

Thymuses from 2- and 4-month-old control rats displayed normal morphology: welldefined cortical regions, densely packed with thymocytes, distinct medullary regions, thin interlobular septa, and small amounts of subcapsular adipose tissue (Figure 1, Panel A). However, in 7- and particularly in 10-monthold rats (Figure 1, Panel B), striking struc- 
tural changes were observed. The thickness of the thymic capsule and septa increased and a substantial quantity of adipose tissue accumulated under the capsule and in the interlobular spaces. The borderline between the cortex and medulla appeared less distinct.

No prominent differences in thymic structure were observed between $\mathrm{Cx}$ and agematched controls.

\section{Computer-assisted morphometric analysis}

Age-associated changes in control rats. The results showed that the relative thymic weight (ratio to $100 \mathrm{~g}$ body weight) of control rats progressively decreased from the age of 30 days to 7 months, and then remained at that level until the age of 10 months (Figure 2, Panel A, b). Furthermore, they revealed that: i) the absolute thymic weight of the same animals was maximal around 2 months of age; ii) between the ages of 2 and 4 months regressive changes took place, so that at 4 months of age thymic

A

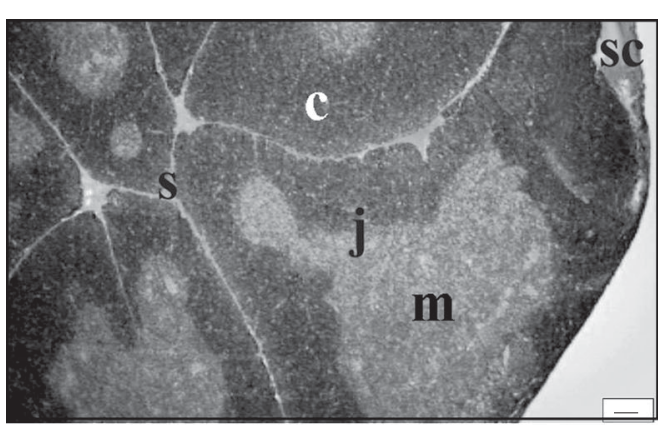

B

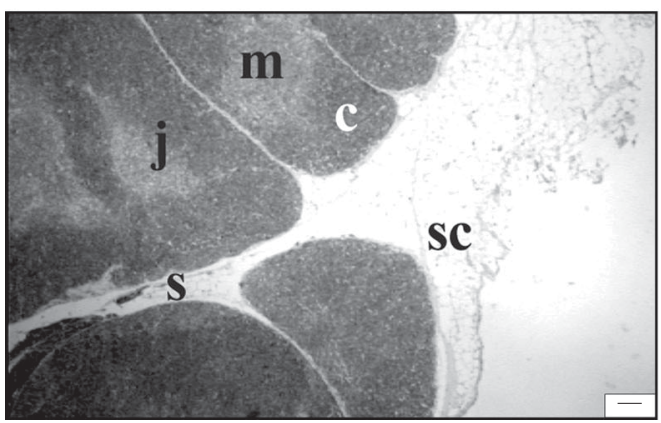

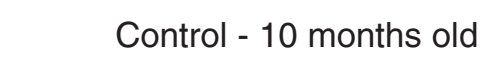

www.bjournal.com.br weight was significantly reduced by an average of 27\% (Figure 2, Panel A, a). This decrease reflected a significant reduction in the size of the thymic cortex (Figure 2, Panel $\mathrm{B}$, a). Absolute thymic weight did not decrease further with age (Figure 2, Panel A, a). However, the volume of thymic cortex continued to shrink until the age of 7 months, and remained at that level for the next 3 months. In spite of the decrease in cortical volume between the ages of 4 and 7 months, the absolute thymic weight remained unaltered due to an increase in the volume of thymic connective/adipose tissue (Figure 2, Panel B, c). Although further enlargement in the volume of this tissue was recorded between the ages of 7 and 10 months, overall thymic weight remained unaffected.

The relative thymic cellularity (ratio to $100 \mathrm{~g}$ body weight) showed a progressive decrease from 30 days up to 10 months of age (Figure 3, Panel A, b). The absolute thymic cellularity exhibited a similar pattern of changes to that shown by absolute organ weight except for an additional significant

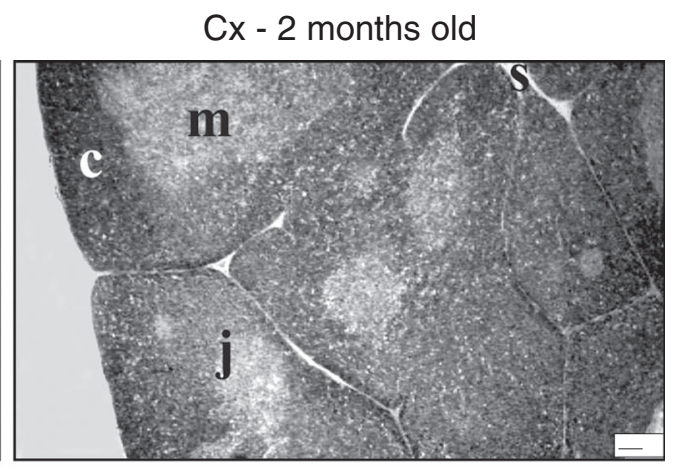

Cx - 10 months old

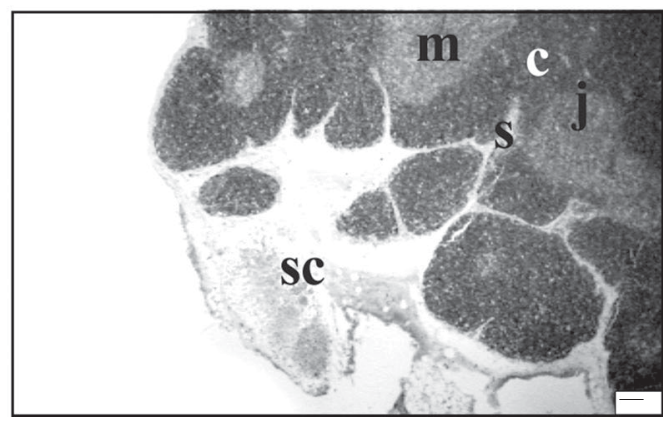

Figure 1. Photomicrographs of thymus sections derived from 2month-old (A) and 10-month-old (B) controls and rats orchidectomized at the age of 30 days (Cx). $\mathrm{c}=$ cortex; $\mathrm{m}=$ medulla; $\mathrm{sc}=$ subcapsular adipose tissue; $\mathrm{j}=$ cortico-medullary junction; $\mathrm{s}=$ septa; H-E staining, 40X. Bar = $100 \mu \mathrm{m}$. 
decrease between the ages of 7 and 10 months (the average thymic cellularity diminished by $66 \%$ from 2 to 10 months of age; Figure 3, Panel A, a). The age-associated changes in thymic cellularity reflected alterations in cortical thymocyte numbers at all time points except for the age of 10 months, when the reduction in organ cellularity was related to a significant decrease in the number of medullary thymocytes due to a decline in their numerical density (Figure 3, Panels B, C).

Effects of orchidectomy. Orchidectomy at the age of 30 days produced a significant increase in relative and absolute thymic weight (Figure 2, Panel A, a,b), as well as in relative and absolute thymocyte number at all time points except for the age of 10 months (Figure 3, Panel A, a,b). At that age neither the relative and absolute thymic weight nor any of the morphometric parameters significantly differed between $\mathrm{Cx}$ and age-matched control rats. Regardless of age $(2,4$, or 7 months), the increase in the thymic weight of $\mathrm{Cx}$ animals reflected an increase in thymic cortical volume (Figure 2, Panel $\mathrm{B}$, a), whereas at the ages of 2 and 4 months increases in medullary volume also contributed to enlargement of the organ (Figure 2, Panel B, b). The significant increase in cortical volume in all groups of $\mathrm{Cx}$ rats correlated with the rise in cellularity in this compartment (Figure 3, Panel B, a). However, only in 4-month-old rats did the increase in medullary volume reflect changes in the number of thymocytes (Figure 3, Panel C, a). In 2-month-old animals the greater volume of this compartment was most likely related to an increase in volume of its non-lymphoid component. Namely, in the medulla of 2month-old rats a significant reduction in thymocyte Nv was found (Figure 3, Panel C, b) and accordingly, in spite of the increased

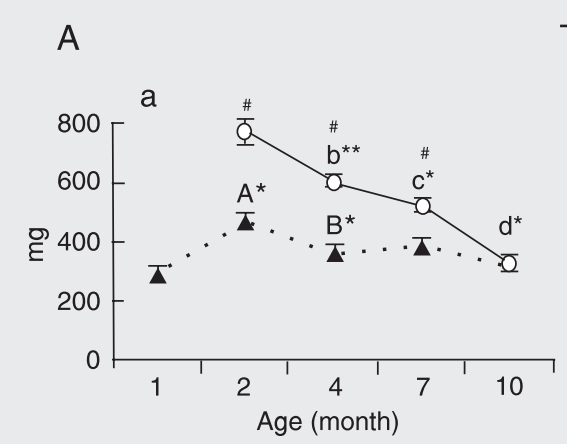

Thymus

B

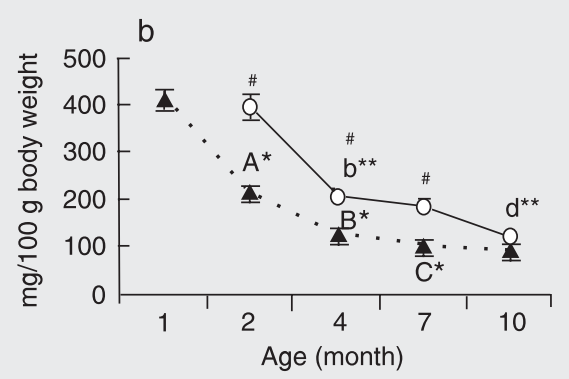

$\mathrm{Sx} O \mathrm{Cx}$
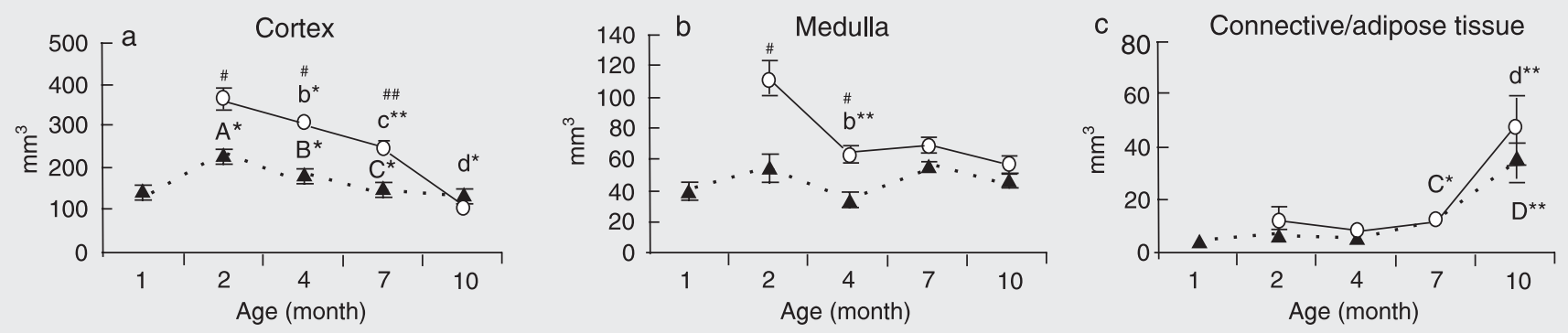

Figure 2. Age-related changes in $A$, absolute thymic weight (a) and relative thymic weight (b), as well as $B$, volume of different thymic compartments: (a) cortex, (b) medulla, and (c) connective/adipose tissue, in rats orchidectomized at the age of 30 days (Cx) and control animals (Sx). Data are reported as means $\pm \mathrm{SEM}$ for $\mathrm{N}=5$. $\mathrm{A}_{2}$-month-old Sx vs 30-day-old Sx; ${ }^{4}$-month-old Sx vs 2-month-old Sx; ${ }^{\mathrm{C}}$-month-old Sx vs 4-month-old Sx; ${ }^{\mathrm{D}} 10$-month-old

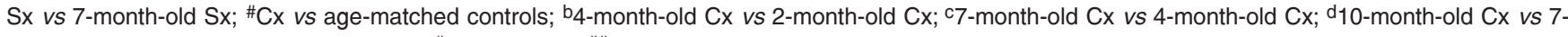
month-old $\mathrm{Cx} .{ }^{*} \mathrm{P}<0.05$ and ${ }^{* *} \mathrm{P}<0.01 ;{ }^{\#} \mathrm{P}<0.05$ and ${ }^{\# \#} \mathrm{P}<0.01$ (Mann-Whitney U-test). 
A

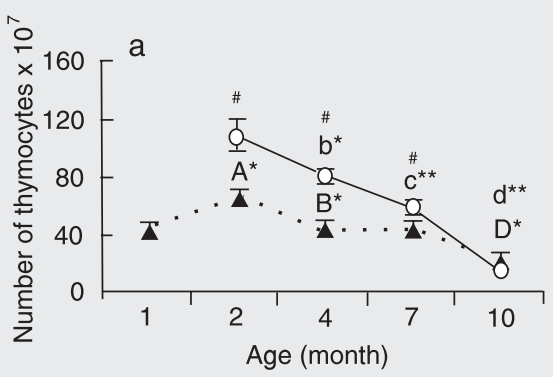

B

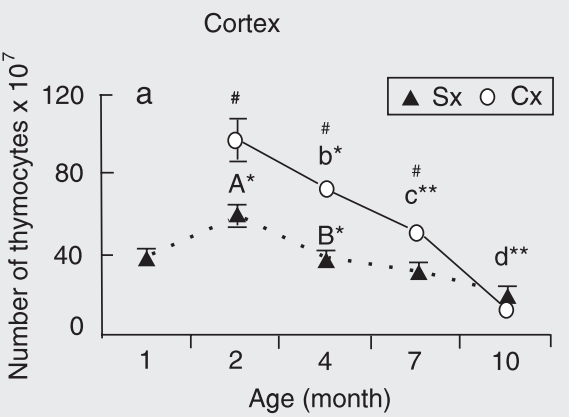

C

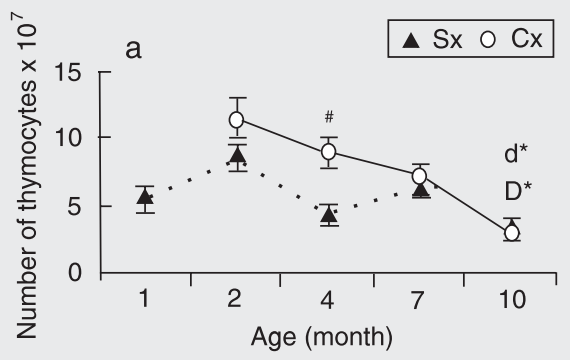

Thymus
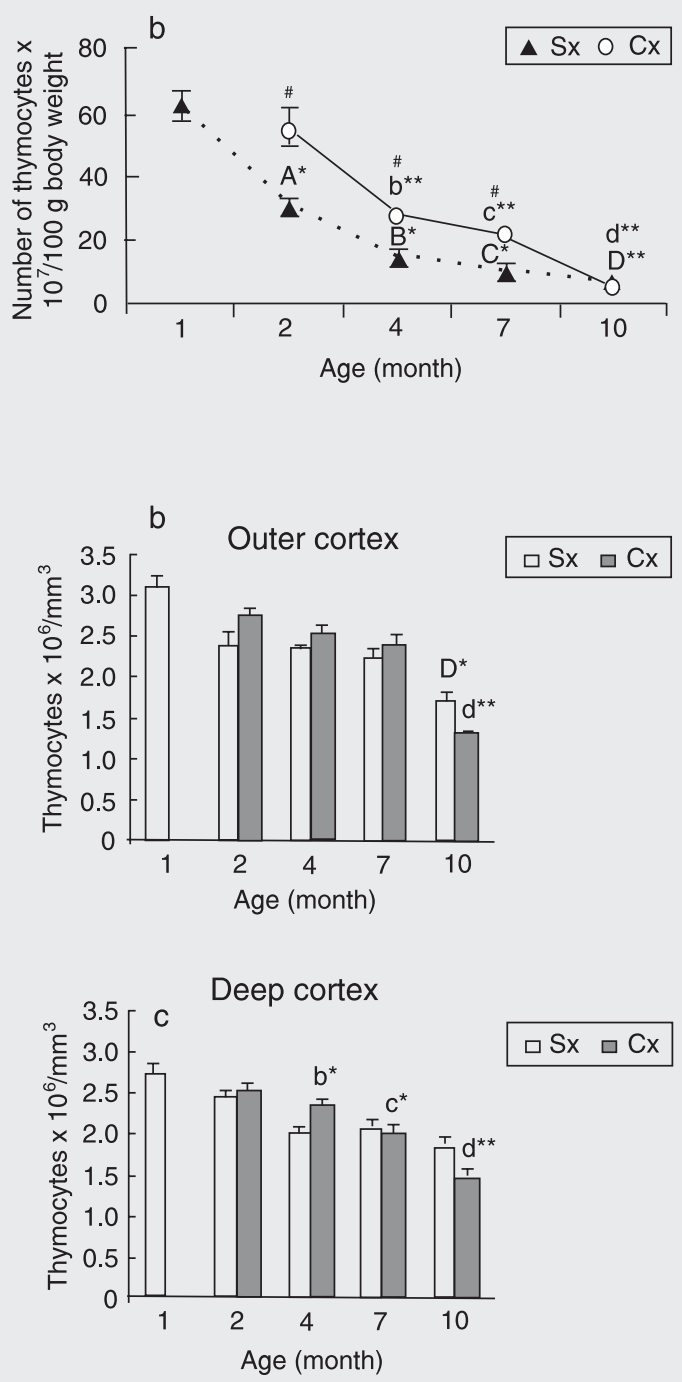

Medulla

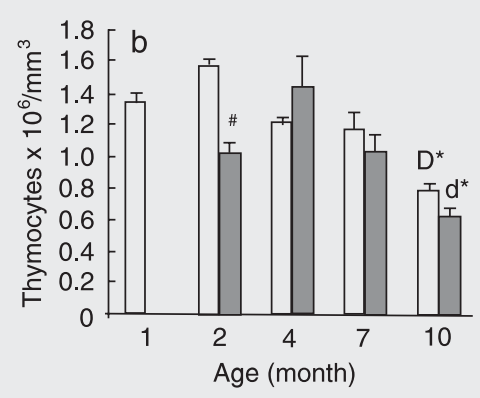

Figure 3. $A$, Age-related changes in the (a) total and (b) relative number of thymocytes, as well as in the number of thymocytes ( $\mathrm{B}$, a and $\mathrm{C}, \mathrm{a})$ and in thymocyte numerical density in the $(B, b, c)$ thymic cortex and $(\mathrm{C}, \mathrm{b})$ medulla in rats orchidectomized at the age of 30 days $(\mathrm{Cx})$ and control animals (Sx). Data are reported as means $\pm S E M$ for $N=5$. $A_{2}$ month-old Sx vs 30-day-old Sx; B 4-month-old Sx vs 2-month-old Sx; $C_{7-m o n t h-o l d ~} S x$ vs 4month-old Sx; D10-month-old Sx vs 7-month-old Sx; "Cx vs agematched controls; b4-month-old Cx vs 2-month-old Cx; ${ }^{c} 7-m o n t h-$ old $\mathrm{Cx}$ vs 4-month-old $\mathrm{Cx}$; $\mathrm{d}_{10}$ month-old $\mathrm{Cx}$ vs 7-month-old Cx. ${ }^{*} \mathrm{P}<0.05$ and ${ }^{* *} \mathrm{P}<0.01$; ${ }^{\#} \mathrm{P}$ $<0.05$ (Mann-Whitney U-test). 
Figure 4. Increased percentage of cycling cells (cells in the S/ G2M phases of the cell cycle) in concanavalin A-stimulated thymocyte cultures from adult rats orchidectomized at the age of 30 days $(\mathrm{Cx})$ as determined by propidium iodide binding to DNA. $A$, Representative overlaid histograms (3-D view) of propidium iodide staining of cells from thymocyte cultures of 1) 2-monthold control rats (Sx), 2) 2-monthold Cx rats, 3) 10-month-old Sx rats, and 4) 10-month-old Cx rats. The arrow points at the subG1 peak that corresponds to apoptotic cells. B, Relative proportion of thymocytes in the S/ G2M phases of the cell cycle in concanavalin A-stimulated thymocyte cultures from control rats (Sx) and rats orchidectomized at the age of 30 days (Cx). Data are reported as means \pm SEM for $\mathrm{N}=5 .{ }^{*} \mathrm{P}<0.05$ and ${ }^{* *} \mathrm{P}<$ 0.01 (Mann-Whitney U-test). volume of the whole compartment, the overall number of lymphoid cells in this compartment remained unaltered (Figure 3, Panel $\mathrm{C}, \mathrm{a})$.

Age-associated changes in Cx rats. Analysis of age-associated changes in $\mathrm{Cx}$ rats demonstrated a progressive decrease in relative and absolute thymic weight between the ages of 2 and 10 months, so that at 10 months neither relative nor absolute thymic weight differed significantly between $\mathrm{Cx}$ and $\mathrm{Sx}$ rats (the average thymic weight was reduced

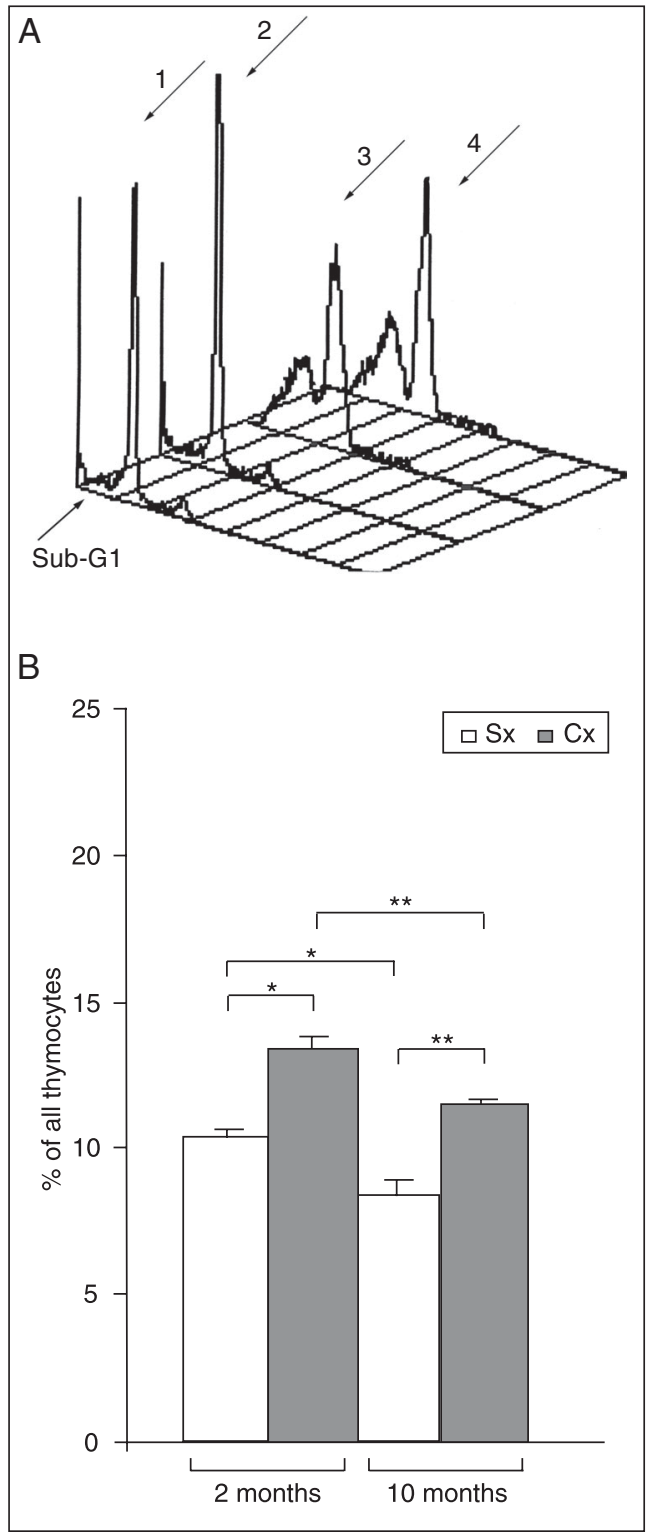

by $57 \%$; Figure 2, Panel A, a,b). This decrease was particularly pronounced between the ages of 4 and 10 months (the average weight was reduced by $45 \%$ ). The reduction of absolute thymic weight mainly reflected the decrease in cortical volume (Figure 2, Panel B, a). A significant decrease in medullary volume (Figure 2, Panel B, b) was found to contribute to the reduction of thymic weight only in 4-month-old rats. In 10-monthold rats a significant enlargement of connective-adipose tissue volume was also recorded (Figure 2, Panel B, c).

In $\mathrm{Cx}$ rats both absolute and relative thymic cellularity also exhibited a progressive decrease between the ages of 2 and 10 months (the average number was reduced by $82 \%$; Figure 3, Panel A, a,b). The changes in thymic cellularity primarily reflected the reduction in number of cortical thymocytes (Figure 3, Panel B, a) due to the progressive loss of these cells in the deep cortex (at all time points) and in the outer cortex (at 10 months of age), which was evident from the reduced Nv of thymocytes in the deep and outer cortical compartments, respectively (Figure 3, Panel B, b,c). Between the ages of 7 and 10 months a significant loss in medullary thymocytes leading to a decrease in $\mathrm{Nv}$ of thymocytes was also recorded (Figure 3 , Panel C, a,b).

\section{Thymocyte proliferation}

To clarify the mechanisms underlying age-dependent alterations in thymic cellularity in ConA+ thymocyte cultures from 2and 10-month-old rats (i.e., at time points when the most striking changes in thymic weight and cellularity were recorded) the percentage of cells in the $\mathrm{S} / \mathrm{G} 2 \mathrm{M}$ active phases of the cell cycle was examined.

The results showed that, irrespective of age, in thymocyte cultures from $\mathrm{Cx}$ rats there was a greater percentage of cells in the $\mathrm{S} /$ G2M phases of the cell cycle than in the corresponding cultures from age-matched 
control rats (Figure 4, Panel B). Furthermore, irrespective of gonadal presence, the percentage of cells in the S/G2M phases of the cell cycle was greater in thymocyte cultures from 2-month-old rats than in corresponding cultures from 10-month-old rats.

It should also be pointed out that the percentage of apoptotic cells forming a flow cytometric peak to the left of the G1 peak (Figure 4, Panel A), which is often referred to as the sub-G1 peak (18), was significantly $(\mathrm{P}<0.01)$ greater in thymocyte cultures from 10-month-old rats $(68.16 \pm 1.4 \%$ in $\mathrm{Sx}$ rats and $75.28 \pm 0.8 \%$ in $\mathrm{Cx}$ rats) than in the corresponding cultures from 2-month-old rats $(25.71 \pm 1.7 \%$ in Sx rats and $22.15 \pm 2.34 \%$ in Cx rats).

\section{Thymocyte apoptosis}

Since not only alterations in thymocyte proliferation, but also in thymocyte apoptosis may affect thymic cellularity, thymocyte sensitivity to apoptotic signaling was estimated at the same time points at which thymocyte proliferative capacity was examined. In thymocyte cultures from 2-month-old $\mathrm{Cx}$ rats the percentage of apoptotic cells was significantly lower than in the age-matched controls. However, on the contrary, in thymocyte cultures from 10-month-old rats the percentage of apoptotic cells was increased in Cx compared to Sx rats (Figure 5, Panel B). Nevertheless, the percentage of apoptotic cells was significantly lower in thymocyte cultures from both $\mathrm{Cx}$ and control 2-monthold rats than in the corresponding cultures from 10-month-old rats (Figure 5, Panel B).

According to the intensity of MC540 fluorescence, on the one hand, and forward scatter, on the other (19), two subsets of apoptotic cells can be distinguished: 1) cells in early apoptosis, and 2) cells in advanced/ late apoptosis (Figure 5, Panel A). Cells in early apoptosis exhibit a high level of MC540 staining, while those in advanced/late apoptosis show lower levels of MC540 staining

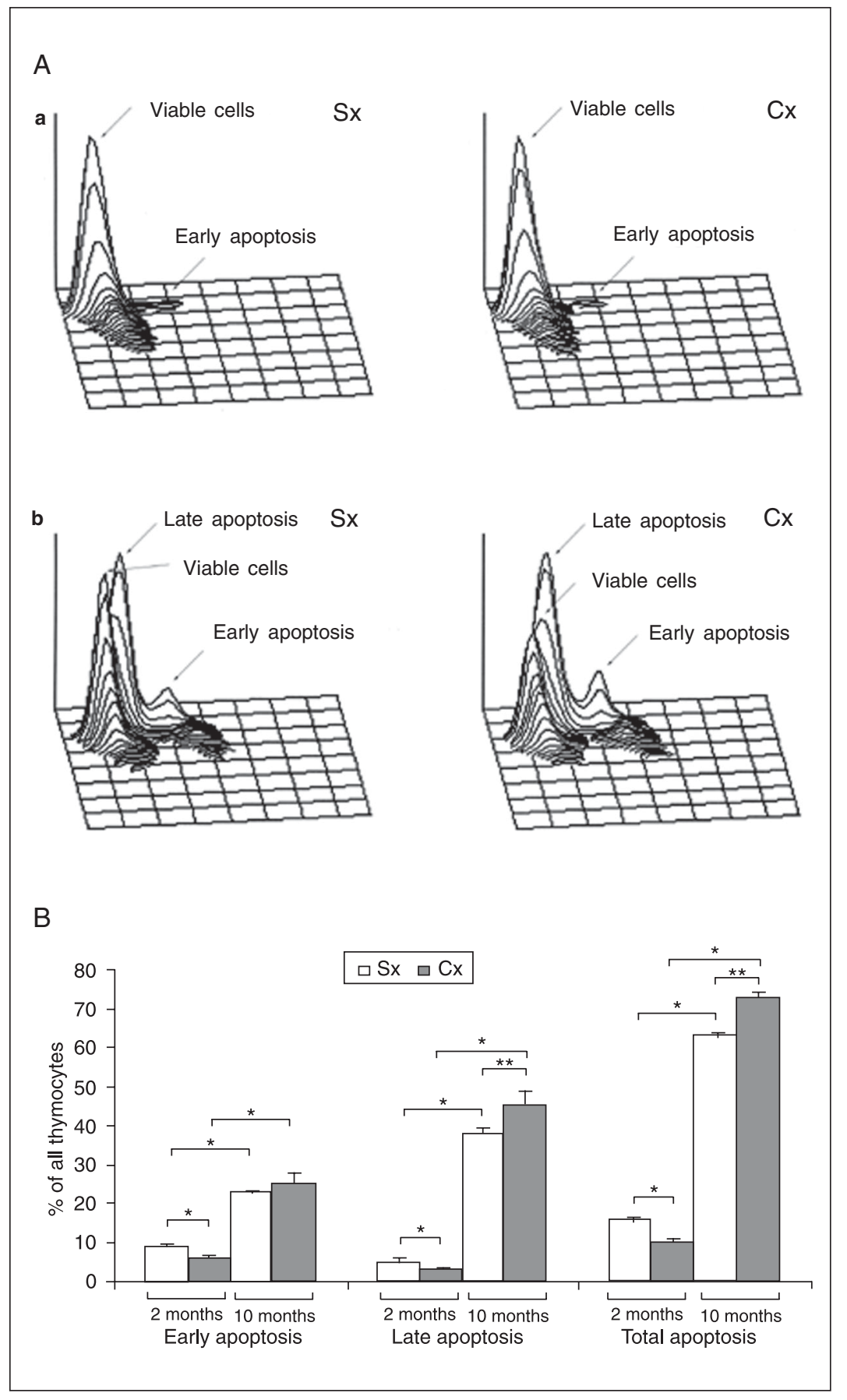

Figure 5. Effects of orchidectomy at the age of 30 days on the frequency of apoptotic cells in 18-h thymocyte cultures. The apoptotic cells were identified by MC540 binding. A, Threedimensional plots of MC540-labeled thymocytes in cultures from (a) 2-month-old and (b) 10-month-old orchidectomized (Cx) and control rats (Sx). According to the intensity of MC540 thymocyte staining and forward scatter, two subsets of cells at distinct phases of apoptosis (early apoptosis and advanced/late apoptosis) were delineated. B, The histogram represents the overall percentage of apoptotic cells and the percentages of cells in early and advanced/late apoptosis in thymocyte cultures from Cx and Sx rats. Data are reported as means \pm SEM for $\mathrm{N}=5$. ${ }^{*} \mathrm{P}<0.05$ and ${ }^{* *} \mathrm{P}<0.01$ (Mann-Whitney U-test). 
and lower forward scatter. In thymocyte cultures from 2-month-old $\mathrm{Cx}$ rats the reduction in the percentage of apoptotic cells reflected decreases in the relative numbers of cells in both the early and late phases of apoptosis (Figure 5, Panel B). On the other hand, the higher percentage of apoptotic cells in 10-month-old $\mathrm{Cx}$ rats compared to age-matched control rats was mainly due to a rise in the proportion of cells in late apoptosis (Figure 5, Panel B).

\section{Discussion}

This study describes the age-related changes in weight, cellularity and morphometric indices of the thymus in peripubertally $\mathrm{Cx}$ and control AO rats. We have confirmed that gonadal hormone removal in the peripubertal period leads to a significant increase in thymic weight and cellularity in young adult rats (8), and have shown that this organ reaches its maximum weight and cellularity one month after surgery. Finally, to the best of our knowledge, for the first time it has been demonstrated that in rats the effects of peripubertal orchidectomy on the thymus are long-lasting but not permanent, i.e., that removal of the testes can only postpone but not prevent the age-related organ atrophy.

The present findings confirm that, after an initial decline in early adulthood, thymic weight in rats (3), as in humans (9) and some other mammals (20), but not in mice (2123), remains unaltered during a relatively long period of time (from 4 to 10 months of age). Although thymic weight in 10-monthold rats did not differ from values for 4- and 7-month-old animals, the total number of thymocytes in both thymic compartments, and hence the overall organ cellularity, were significantly reduced reflecting, most likely, an age-associated increase in thymocyte sensitivity to apoptotic stimuli followed by decreased responsiveness to proliferative signaling. This assumption emerged from our findings obtained with thymocyte cultures, which are fully consistent with previously reported data on cell sensitivity to apoptotic and proliferative signaling in thymocyte cultures from aged Wistar rats (14). The ageassociated expansion of connective-adipose tissue described here, which compensates for the loss of lymphoid tissue, has also been observed in aged male Wistar rats (24). Therefore, these results support our previous findings that the overall thymic weight of aging rats does not correlate with the amount of functional lymphoid thymic tissue (24).

Orchidectomy at the age of 30 days produced a pronounced increase in both thymic weight and cellularity at all examined points from 1 to 6 months post-castration, but at 9 months post-castration, none of these parameters significantly differed between $\mathrm{Cx}$ and age-matched controls. At all time points examined between 1 and 6 months postcastration both the size and the cellularity of the cortical compartment were increased, while these parameters of the thymic medulla were augmented only at 3 months after castration. The increases in thymic weight and cellularity evoked by orchidectomy may be associated with: i) improved precursor generation in the bone marrow (25), ii) alterations in the thymic microenvironment resulting in more efficient attraction of thymocyte progenitors $(26)$, iii) increased thymocyte sensitivity to proliferative stimuli (27), and iv) reduced thymocyte sensitivity to apoptotic stimuli (28). Our results showed that 1 month after surgery (when thymic weight and cellularity were at their maximum), the increase in the overall thymocyte number most probably reflected an increased thymocyte sensitivity to proliferative stimuli on the one hand, and reduced sensitivity to apoptotic stimuli on the other. In agreement, there are data showing that androgens inhibit thymocyte proliferation (29) and accelerate thymocyte elimination via apoptosis $(28,30)$. The present findings also demonstrate that 9 months after orchidectomy nei- 
ther thymic weight nor any estimated morphometric parameter significantly differed from those in age-matched controls. This is in keeping with the very recent conclusion of Min and colleagues (10) that the orchidectomy-induced effect on thymic weight in mice is of limited duration. In other words, our results indicate that removal of the testes in rats, as in mice (10), postpones age-associated thymic involution, but does not prevent it completely. Furthermore, our results also indicate that a new balance between thymocyte apoptosis and proliferation was established in these animals. Differently from thymocyte cultures from 2-month-old rats, the percentage of apoptotic cells was significantly greater in thymocyte cultures from $\mathrm{Cx}$ rats compared with age-matched controls. Moreover, although the proportion of cells in the S/G2M phases of the cell cycle was significantly greater in ConA+ thymocyte cultures from 10-month-old $\mathrm{Cx}$ rats than in those from age-matched Sx rats, it was significantly less than in thymocyte cultures from 2-month-old Cx rats. A compensatory intrathymic increase in androgen production sufficient to cause accelerated thymocyte apoptosis, but still not sufficient to prevent proliferation of these cells may be assumed to explain these findings. In favor of this hypothesis are data suggesting that both lymphocytes (31) and TEC may produce steroid hormones (32). Furthermore, analysis of thymocyte differentiation/maturation under the same experimental conditions showed that alterations in the relative proportion of thymocytes (delineated by expression of $\mathrm{CD} 4, \mathrm{CD} 8$ and T-cell receptor) become quantitatively more pronounced with duration of gonadal deprivation (8). Thus, the present findings, taken together with those previously published (8), indicate that, although 9 months after orchidectomy a new balance between the main thymic homeostatic processes (i.e., apoptosis and proliferation) is established, so that thymic cellularity does not significantly differ between
Cx and control rats, the thymocyte differentiation kinetics remains substantially altered, producing an accumulation of cells at one maturational step and depletion at the subsequent downstream steps. Moreover, the ageassociated decline in thymic weight and cellularity in Cx rats was even more pronounced than that in non-operated animals and, in contrast to control rats, both thymic weight and cellularity exhibited a progressive reduction between the ages of 2 and 10 months. This suggests that: i) age-associated thymic atrophy, which is initially sensitive to circulating androgen levels, becomes independent of them with progression of the process and ii) the kinetics of age-associated thymic involution depends on the presence of circulating gonadal hormones.

To explain the limited duration of effects induced by gonadal hormone ablation on age-related thymic atrophy several possibilities should be taken into consideration. First, as mentioned above, lymphocytes themselves have been shown to produce androgens (31), so that a post-castrational compensatory increase in intrathymic androgen production from re-entrant and/or developing lymphocytes cannot be ruled out. Furthermore, since TEC possess a complete intracellular machinery for the synthesis of steroid hormones, and since they are supposed to produce these hormones (32), a compensatory increase in TEC androgen production should also be taken into account. In support of the previous assumption are data indicating that: i) the ratio of non-lymphoid to lymphoid component volume was increased in the thymic medulla of rats subjected to gonadal deprivation for 9 months and ii) androgens influence TEC proliferation in a biphasic mode, reducing their proliferation at greater concentrations than optimal in a dose-dependent manner, and increasing it at less than optimal concentrations (33). Second, compensatory production of adrenal androgens should be taken into account. However, it has been shown 
that the adrenals of rats and mice do not synthesize androgens (34), so that in these animals orchidectomy is not followed by increased serum levels of either dehydroepiandrosterone or testosterone (35) and this hypothesis should be rejected. Third, it may be assumed that thymic involution is mediated by distinct mechanisms acting during different periods of ontogenesis or that some other, non-androgen-mediated mechanism (e.g., growth hormone-dependent) (36), may take over the androgen role in long-term androgen-deprived rats. At present there are no data available to strongly support this hypothesis. Finally, it may also be hypothesized that gonadal hormones are important in the initial phases of thymic regression, while later accumulating age-related functional or structural defects (37) are responsible for the maintenance/progression of thymic involution. In agreement with this hypothesis are data showing that: i) normal, agerelated androgen depletion (38) exerts no beneficial effect on the aged thymus (39), and ii) castration of old rats does not restore thymic weight to the level found in young animals (40).

In conclusion, this study has clearly shown that gonadal ablation cannot prevent thymic involution, suggesting that even if circulating gonadal hormones are predominantly responsible for the initiation of ageassociated thymic involution, maintenance of their circulating levels is not necessary to secure maintenance/progression of the ageassociated regressive thymic changes. To further clarify the role of androgens in the initiation, and particularly in the maintenance/progression of age-related thymic atrophy, experiments addressing not only thymic effects evoked by long-lasting withdrawal of circulating androgens, but also those induced by blockade of intrathymically synthesized/released androgen action, should be undertaken.

\section{References}

1. Grossman CJ. Interactions between the gonadal steroids and the immune system. Science 1985; 227: 257-261.

2. Bodey B, Bodey B Jr, Siegal SE, Kaiser HE. Involution of the mammalian thymus, one of the leading regulators of aging. In Vivo 1997; 11: 421-440.

3. Quaglino D, Capri M, Bergamini G, Euclidi L, Zecca L, Ronchetti IP. Age-dependent remodeling of rat thymus. Morphological and cytofluorimetric analysis from birth up to one year of age. Eur $\mathrm{J}$ Cell Biol 1998; 76: 156-166.

4. Pawelec G, Effros RB, Caruso C, Remarque E, Barnett Y, Solana R. T cells and aging (update February 1999). Front Biosci 1999; 4: D216-D269.

5. Viselli SM, Olsen NJ, Shults K, Steizer G, Kovacs WJ. Immunochemical and flow cytometric analysis of androgen receptor expression in thymocytes. Mol Cell Endocrinol 1995; 109: $19-26$.

6. Olsen NJ, Olson G, Viselli SM, Gu X, Kovacs WJ. Androgen receptors in thymic epithelium modulate thymus size and thymocyte development. Endocrinology 2001; 142: 1278-1283.

7. Kendall MD, Fitzpatrick FT, Greenstein BD, Khoylou F, Safieh B, Hamblin A. Reversal of ageing changes in the thymus of rats by chemical or surgical castration. Cell Tissue Res 1990; 261: 555564.

8. Leposavić G, Pejcić-Karapetrović B, Kosec D. Alterations in thymopoiesis in intact and peripubertally orchidectomized adult rats of different age. Mech Ageing Dev 2002; 123: 401-411.
9. Steinmann GG, Klaus B, Muller-Hermelink HK. The involution of the ageing human thymic epithelium is independent of puberty. A morphometric study. Scand J Immunol 1985; 22: 563-575.

10. Min H, Montecino-Rodriguez E, Dorshkind K. Reassessing the role of growth hormone and sex steroids in thymic involution. Clin Immunol 2006; 118: 117-123.

11. Plećaš-Solarović B, Lalić Lj, Leposavić G. Age-dependent morphometrical changes in the thymus of male propranolol-treated rats. Ann Anat 2004; 186: 141-147.

12. Lind EF, Prockop SE, Porritt HE, Petrie HT. Mapping precursor movement through the postnatal thymus reveals specific microenvironments supporting defined stages of early lymphoid development. J Exp Med 2001; 194: 127-134.

13. Kendall MD, Al-Shawaf AA. Innervation of the rat thymus gland. Brain Behav Immun 1991; 5: 9-28.

14. Leposavić G, Pešić V, Kosec D, Radojević K, Arsenović-Ranin N, Pilipović I, et al. Age-associated changes in CD90 expression on thymocytes and in TCR-dependent stages of thymocyte maturation in male rats. Exp Gerontol 2006; 41: 574-589.

15. Kamath $A B$, Nagarkatti PS, Nagarkatti M. Characterization of phenotypic alterations induced by 2,3,7,8-tetrachlorodibenzo-p-dioxin on thymocytes in vivo and its effect on apoptosis. Toxicol Appl Pharmacol 1998; 150: 117-124.

16. Laakko T, King L, Fraker P. Versatility of merocyanine 540 for the flow cytometric detection of apoptosis in human and murine cells. $J$ 
Immunol Methods 2002; 261: 129-139.

17. Mower DA Jr, Peckham DW, Illera VA, Fishbaugh JK, Stunz LL, Ashman RF. Decreased membrane phospholipid packing and decreased cell size precede DNA cleavage in mature mouse B cell apoptosis. J Immunol 1994; 152: 4832-4842.

18. Ormerod MG. Investigating the relationship between the cell cycle and apoptosis using flow cytometry. J Immunol Methods 2002; 265: 73-80.

19. Cohen JJ. Programmed cell death in the immune system. Adv Immunol 1991; 50: 55-85.

20. Hale LP, Clark AG, Li J, Greer PK, Byers Kraus V. Age-related thymic atrophy in the guinea pig. Dev Comp Immunol 2001; 25: 509518.

21. Nabarra B, Andrianarison I. Ultrastructural study of thymic microenvironment involution in aging mice. Exp Gerontol 1996; 31: 489-506.

22. Lau LL, Spain LM. Altered aging-related thymic involution in T cell receptor transgenic, MHC-deficient, and CD4-deficient mice. Mech Ageing Dev 2000; 114: 101-121.

23. Li L, Hsu HC, Grizzle WE, Stockard CR, Ho KJ, Lott P, et al. Cellular mechanism of thymic involution. Scand J Immunol 2003; 57: 410422.

24. Plećaš-Solarović B, Pešić V, Radojević K, Leposavić G. Morphometrical characteristics of age-associated changes in the thymus of old male Wistar rats. Anat Histol Embryol 2006; 35: 380-386.

25. Ishimi Y, Yoshida M, Wakimoto S, Wu J, Chiba H, Wang X, et al. Genistein, a soybean isoflavone, affects bone marrow lymphopoiesis and prevents bone loss in castrated male mice. Bone 2002; 31 : 180-185.

26. Gill J, Malin M, Sutherland J, Gray D, Hollander G, Boyd R. Thymic generation and regeneration. Immunol Rev 2003; 195: 28-50.

27. Windmill KF, Lee VW. Influences of surgical castration on the thymus of male rats. J Reprod Immunol 1999; 44: 29-39.

28. Olsen NJ, Viselli SM, Fan J, Kovacs WJ. Androgens accelerate thymocyte apoptosis. Endocrinology 1998; 139: 748-752.

29. Olsen NJ, Viselli SM, Shults K, Stelzer G, Kovacs WJ. Induction of immature thymocyte proliferation after castration of normal male mice. Endocrinology 1994; 134: 107-113.

30. Guevara Patino JA, Marino MW, Ivanov VN, Nikolich-Zugich J. Sex steroids induce apoptosis of CD8+CD4+ double-positive thymocytes via TNF-alpha. Eur J Immunol 2000; 30: 2586-2592.

31. Zhou Z, Shackleton CH, Pahwa S, White PC, Speiser PW. Prominent sex steroid metabolism in human lymphocytes. Mol Cell Endocrinol 1998; 138: 61-69.

32. Khosla S, Ovalle WK. Morphology and distribution of cystic cavities in the normal murine thymus. Cell Tissue Res 1986; 246: 531-542.

33. Sakabe K, Kawashima I, Urano R, Seiki K, Itoh T. Effects of sex steroids on the proliferation of thymic epithelial cells in a culture model: a role of protein kinase C. Immunol Cell Biol 1994; 72: 193199.

34. van Weerden WM, Bierings HG, van Steenbrugge GJ, de Jong FH, Schroder $\mathrm{FH}$. Adrenal glands of mouse and rat do not synthesize androgens. Life Sci 1992; 50: 857-861.

35. Belanger B, Belanger A, Labrie F, Dupont A, Cusan L, Monfette G. Comparison of residual C-19 steroids in plasma and prostatic tissue of human, rat and guinea pig after castration: unique importance of extratesticular androgens in men. J Steroid Biochem 1989; 32: 695698.

36. Kelley KW, Brief S, Westly HJ, Novakofski J, Bechtel PJ, Simon J, et al. GH3 pituitary adenoma cells can reverse thymic aging in rats. Proc Natl Acad Sci USA 1986; 83: 5663-5667.

37. Montecino-Rodriguez E, Clark R, Dorshkind K. Effects of insulin-like growth factor administration and bone marrow transplantation on thymopoiesis in aged mice. Endocrinology 1998; 139: 4120-4126.

38. Kaler LW, Neaves WB. The androgen status of aging male rats. Endocrinology 1981; 108: 712-719.

39. Taub DD, Longo DL. Insights into thymic aging and regeneration. Immunol Rev 2005; 205: 72-93.

40. Utsuyama M, Hirokawa K. Hypertrophy of the thymus and restoration of immune functions in mice and rats by gonadectomy. Mech Ageing Dev 1989; 47: 175-185. 


\section{Rio de Janeiro Cell Bank}

\section{Human and Animal Cell Biotechnology Services}

- Deposit and Distribution of Cell Lines

- Cell Cryopreservation

- Development and Distribution of Human and Animal Primary Cell Cultures

- Development of New Monoclonal Antibody Secreting Hybridomas

- Monoclonal Antibodies, in Cell Culture Supernatants or Purified

- Identification of Microbiological Contaminants

- Decontamination of Cell Lines

- Cytotoxicity Tests

- Characterization of Cell Lines

- Training in "in vitro" Culture of Human and Animal Cells

- Consultation Assistance in Cell Culture

\section{Contact us for specific services not listed above}

Banco de Células do Rio de Janeiro - BCRJ Rio de Janeiro Cell Bank

Associação Técnico Científica Paul Ehrlich Hospital Universitário Clementino Fraga Filho/4andar/Sala 4 a 9 Programa Avançado de Biologia Celular Aplicada à Medicina - PABCAM Universidade Federal do Rio de Janeiro - UFRJ, Cidade Universitária Ilha do Fundão, CEP 21941-097 Rio de Janeiro, RJ Tel: (21) 2562.24.67/2562.64.84/2562.64.83 bcrj@hucff.ufrj.br/http://www.bcrj.hucff.ufrj.br/ 